\title{
Juicio, relación múltiple y la teoría cognitivista de las proposiciones
}

\section{[Judgement, Multiple Relation, and the Cognitive Theory of Propositions]}

\author{
JAVIER VIDAL \\ Universidad de Concepción \\ Chile \\ fravidal@udec.cl
}

\begin{abstract}
Resumen: Según la teoría cognitivista, las proposiciones son tipos de actos predicativos, y un agente lleva a cabo una predicación cada vez que juzga o asevera algo. Ahora bien, la versión predominante de dicha teoría establece que el juicio es una actitud cognitiva hacia un contenido proposicional genuino, lo que significa que se lo entiende como una relación dual entre un agente y una proposición. Tras mostrar algunos problemas con esta versión, argumentaré a favor de una teoría cognitivista de las proposiciones en la que el juicio se entiende como una relación múltiple entre un agente y entidades del mundo tales como objetos, propiedades y relaciones.
\end{abstract}

Palabras clave: tipos de acto; relaciones de ejemplificación; representación; predicación; Hanks

\begin{abstract}
According to cognitive theory, propositions are types of predicative acts, and an agent performs a predication every time she judges or asserts something. Now, the prevailing version of such a theory states that judgement is a cognitive attitude toward a genuine propositional content, which means that it is seen as a dual relation between an agent and a proposition. After showing some troubles with this version, I will argue for a cognitive theory of propositions in which judgement is seen as a multiple relation between an agent and worldly entities such as objects, properties and relations.
\end{abstract}

Keywords: act-types; tokening relations; representation; predication; Hanks

Una proposición es el contenido de una actitud psicológica o de un acto de habla, o lo que expresa una oración. Se suele suponer entonces que, por ejemplo, la proposición de que Lara es gris es lo que puedo juzgar, o aseverar, mediante una emisión de la oración "Lara es gris". Para distinguirla de la oración, me referiré a esa proposición con la expresión "<Lara es gris $>$ ". En este artículo, consideraré sólo las proposiciones de naturaleza declarativa, es decir, las proposiciones que pueden ser el contenido de actitudes cognitivas, como diré, y de actos de habla cuyo objetivo es representar cómo son las cosas en el mundo. Se trataría en principio de actitudes como concebir, juzgar, dudar y negar, entre otras, y actos de habla como aseverar.

Diánoia, vol. 66, no. 87 (noviembre de 2021-abril de 2022): pp. 45-74 • ISSN: 1870-4913 DOI: http://doi.org/10.22201/iifs.18704913e.2021.87.1842 
Se sabe que en una época Bertrand Russell defendió una teoría de las proposiciones según la cual una proposición es una entidad estructurada, constituida a su vez por ciertas entidades del mundo como objetos, propiedades y relaciones. Por ejemplo, la proposición < Lara es gris > sería el estado de cosas cuyos constituyentes son la gata Lara y la propiedad de ser gris. Pero después Russell sostuvo que las expresiones lingüísticas para hablar de las proposiciones, como "que Lara es gris", son "símbolos incompletos", lo que significa que tales expresiones no figurarían en un análisis lógico completo de una oración como "Coetzee juzgó que Lara es gris". Ésta era la contraparte lingüística de una teoría del juicio, o de la creencia, según la cual un juicio no debía entenderse ya como una relación dual entre un agente y una proposición. Según la nueva teoría del juicio sin proposiciones, un juicio se entenderá ahora como una relación entre un agente y las entidades del mundo que antes constituían una proposición russelliana estructurada. Se habla entonces de una teoría del juicio como una relación múltiple, en el sentido de que dicha relación involucra al menos tres entidades, a saber, un agente, un objeto y una propiedad. Así, el juicio que reporta una emisión de "Coetzee juzgó que Lara es gris" no consiste en una relación dual entre Coetzee y la proposición <Lara es gris >, sino en una relación múltiple entre Coetzee, la gata Lara y el color gris (Russell 1912, cap. 12; Russell 1913, pp. 105-118). ${ }^{1}$

No es mi propósito explorar aquí la teoría del juicio como relación múltiple que propuso Russell. Más bien, me ocuparé de defender la articulación entre una teoría del juicio como relación múltiple y una teoría cognitivista de las proposiciones, tal y como aparece formulada recientemente en Soames 2014a y Soames 2015, así como en Hanks 2011, Hanks 2013 y Hanks 2015. Por lo tanto, no se trata, en conformidad con Russell, de cuestionar el lenguaje sobre las proposiciones ni las proposiciones mismas como entidades genuinas, pues justamente propondré que una teoría cognitivista, una teoría comprometida con la indispensabilidad de las proposiciones, debería considerar el juicio como una relación múltiple. Primero expondré las ideas fundamentales de una teoría cognitivista (sección 1). A continuación, mostraré cómo la concepción del juicio como una relación dual, según la versión de Hanks, enfrenta problemas serios (sección 2). Procederé entonces a argumentar, por un lado, que el juicio es una relación múltiple y, por otro lado, que las demás actitudes cognitivas deben entenderse como

\footnotetext{
${ }^{1}$ Para ser más precisos, en el manuscrito de 1913 la actitud cognitiva básica es la comprensión (understanding), no el juicio, y además Russell introduce la forma lógica en la explicación.
} 
relaciones duales con proposiciones (sección 3). Mostraré, por último, que el análisis estándar de los reportes mediante los cuales atribuimos un juicio a un agente no plantea ninguna dificultad para una teoría del juicio como relación múltiple (sección 4).

\section{La teoría cognitivista de las proposiciones}

En la medida en que una proposición declarativa es el contenido tanto de las actitudes cognitivas como de ciertos actos de habla, parece tener propiedades representacionales: la proposición <Lara es gris $>$ representa la gata Lara como gris. En consecuencia, las proposiciones tienen condiciones de verdad (y, por lo tanto, son portadoras de la verdad o la falsedad): la proposición <Lara es gris > es verdadera si y sólo si Lara es gris (Soames 2014a, pp. 91-95; Hanks 2015, pp. 3-4). Ahora bien, las teorías más aceptadas, según las cuales las proposiciones son conjuntos de mundos posibles o son estados de cosas, se enfrentan precisamente al problema de la representación, pues, ¿qué podría hacer que el conjunto de mundos posibles en los que Lara es gris o el estado de cosas cuyos constituyentes son Lara y el color gris fuesen una representación de Lara como gris? Por esta razón, una teoría cognitivista se propone explicar el carácter representacional de las proposiciones en términos de los actos de predicación que los agentes pueden llevar a cabo (en el pensamiento o en el lenguaje). De entrada, la idea es que un agente se representa a Lara como gris porque predica ser gris de Lara. Pero distintos agentes pueden llevar a cabo el mismo tipo de acto predicativo y tener actitudes cognitivas o realizar actos de habla con el mismo contenido proposicional. Entonces, la proposición <Lara es gris > es el tipo de acto cognitivo que consiste en predicar ser gris de Lara, y el agente que en una ocasión particular predica ser gris de Lara produce un ejemplar (token) de esa proposición (Soames 2014a, pp. 95-97; Hanks 2015, pp. 20-24, 73-80). ${ }^{2}$

\footnotetext{
${ }^{2}$ Por mor de la simplicidad, no utilizaré el complejo simbolismo de Hanks 2015 (pp. 25-26): por ejemplo, la proposición de que Lara es gris sería $+<$ Lara, SER GRIS>, donde Lara es el acto de referirse a Lara, SER GRIS es el acto de expresar la propiedad de ser gris y $\vdash$ es el acto de predicar ser gris de Lara. En realidad prefiero destacar, como Soames, sólo el acto predicativo mismo, y por eso hablaré de la proposición < Lara es gris > del mismo modo que Soames hablaría de la proposición que Lara es gris. Desde luego que hay aquí cuestiones que pueden no ser sólo terminológicas: a diferencia de Hanks sostengo, como parece hacer Soames 2014a (pp. 101-102, 120), que en algún sentido Lara y ser gris son "constituyentes" de esa proposición. Pero la proposición no es un estado de cosas del mundo, pues el acto
} 
Una teoría cognitivista de las proposiciones debe dar cuenta de las distintas actitudes cognitivas como distintas actitudes hacia la proposición que es su objeto intencional. Puesto que una proposición es un tipo de acto predicativo, la teoría tendrá que explicar cuál es la conexión entre las actitudes cognitivas y esos actos cognitivos que son los actos de predicación. A este respecto, tanto Hanks como Soames sostienen que las distintas actitudes cognitivas son o involucran actos de predicación. Esto significa que, por ejemplo, mi juicio particular de que Lara es gris involucra el acto de predicar ser gris de Lara y, en este sentido, es, como todas las otras actitudes cognitivas hacia la proposición <Lara es gris $>$, un ejemplar de esa misma proposición. Así, Hanks habla de las distintas actitudes cognitivas como relaciones de ejemplificación (tokening relations) con una u otra proposición (Hanks 2015, p. 161).

Sin embargo, si las distintas actitudes cognitivas hacia una determinada proposición consisten en llevar a cabo el mismo tipo de acto predicativo, entonces debe haber otros rasgos que permitan diferenciar las actitudes cognitivas entre sí. La vía que tanto Hanks como Soames exploran apela primero a una actitud cognitiva básica que no incluye ningún rasgo adicional. En el caso de Soames, la actitud cognitiva básica es concebir o considerar (entertain) una proposición sin llegar a juzgarla, y establece que concebir una proposición es simplemente llevar a cabo el correspondiente acto de predicación. Así, juzgar una proposición añade al acto neutro de predicación el rasgo de afirmarla o aseverarla (y las demás actitudes cognitivas introducirían distintos rasgos adicionales) (Soames 2014b, pp. 228-230). En el caso de Hanks, la actitud básica es juzgar una proposición, lo que implica que la predicación no es neutra, sino que tiene fuerza afirmativa o asertórica. Por lo tanto, es necesario explicar en qué sentido concebir una proposición sin juzgarla consiste en llevar a cabo un acto de predicación. Para Hanks se trataría de que, por ejemplo, un agente predica ser gris de Lara con la respectiva fuerza afirmativa, y el contexto cancela dicha fuerza con el resultado de que finalmente el agente sólo concibió la proposición < Lara es gris $>$. Aquí el rasgo adicional es la cancelación, pues concebir una proposición no significa hacer algo menos que juzgarla, sino justamente hacer algo más: es predicar más cancelar la fuerza afirmativa de esa predicación (Hanks 2015, pp. 33-41, 93-94). ${ }^{3}$

predicativo es un acto mental. Ahora bien, esta diferencia entre Hanks y Soames no afecta de manera significativa el desarrollo del artículo, y por ello usaré el simbolismo más sencillo.

${ }^{3}$ Hanks no habla de concebir una proposición, lo que según él ya implicaría aceptar la distinción entre la fuerza y el contenido (o la predicación) que Soames esta- 
Como Hanks, defenderé una teoría cognitivista de las proposiciones según la cual el juicio es la actitud cognitiva básica. Puesto que argumentaré que mi versión es preferible, entre otras cosas porque ofrece una explicación más económica de la diferencia entre concebir y juzgar una proposición, me detengo brevemente en ello. Uno de los desafíos para cualquier explicación de dicha diferencia es dar cuenta de los juicios que contienen proposiciones no aseveradas como objetos de predicación. En efecto, supongamos que, según una teoría cognitivista, juzgar o aseverar (mediante una oración) la proposición disyuntiva $<p$ o $q>$ consiste en predicar la relación de disyunción de las proposiciones $<p>$ y $<q>$. Como está claro, un agente que realiza ese juicio no juzga o asevera ninguna de estas últimas proposiciones. Podríamos decir entonces que sólo concibe dichas proposiciones, y que así aparecen como proposiciones no aseveradas en el juicio disyuntivo. Pues bien, la explicación que Hanks ofrece de este proceso introduce dos operaciones. Sostiene, primero, que el agente lleva a cabo los actos de predicación que corresponden a $\langle p>\mathrm{y}\langle q>$, pero que la conectiva oracional "o", o su equivalente mental en el juicio, crea un contexto de cancelación de la fuerza afirmativa con el resultado de que el agente finalmente sólo concibe dichas proposiciones. Sin embargo, es necesario explicar también cómo esas proposiciones se convierten en objetos de predicación en el juicio disyuntivo. Supongamos que una de esas proposiciones es $<$ Lara es gris $>$. La propuesta de Hanks es que el mero hecho de predicar ser gris de Lara produce un cambio de objetivo (target-shifting) por el que el acto de predicar, que ahora es el acto de predicar la disyunción, se dirige a la proposición <Lara es gris > (Hanks 2015, pp. 98-108). Como mostraré, mi propuesta es mejor porque no requiere introducir una operación de cancelación ni una operación de cambio de objetivo.

\section{Los problemas con el juicio como relación dual}

Acabamos de ver que, en una teoría cognitivista de las proposiciones, la actitud cognitiva básica (así como cualquier otra actitud cognitiva) es, por un lado, una relación hacia una proposición como su objeto intencional y es, por otro lado, una relación de ejemplificación con esa misma proposición como un tipo de predicación. Parece así que, para Hanks, mi juicio de que Lara es gris tiene como objeto la proposición < Lara es

blece, sino que habla de un acto de predicación que, en cierto contexto, no cuenta como un juicio o una aserción. No tendré presente esta diferencia, pues Hanks aún debería decirnos cuál es entonces la actitud cognitiva hacia una proposición que, en un contexto cancelatorio, acompaña a la predicación. 
gris $>$ y a la vez es un ejemplar de esa proposición como el tipo de acto que consiste en predicar ser gris de Lara. A continuación, discutiré la propuesta de Hanks a este respecto no sólo porque estoy de acuerdo en que el juicio es la actitud cognitiva básica, como propondré en la próxima sección, sino también porque me parece que él formula mejor que Soames esa condición doble.

Téngase en cuenta que, según esto, mi juicio sería tanto una relación dual entre yo y la proposición <Lara es gris > como, en términos de predicación, una relación múltiple entre yo, la gata Lara y la propiedad de ser gris. En efecto, el acto que yo llevo a cabo al predicar ser gris de Lara consiste, como tal predicación, en una relación entre esas tres entidades, no en una relación entre dos entidades una de las cuales es una proposición. El problema ahora es que no tiene sentido decir que, como tipos, la relación dual entre un agente y la proposición $<$ Lara es gris > es la relación múltiple entre un agente, Lara y ser gris. ¿Qué podría significar que estar en una relación con cierta entidad, una proposición, es lo mismo que estar en una relación con otras entidades, un objeto y una propiedad? Hanks 2015 (p. 28) introduce entonces una distinción entre el juicio y la predicación como tipos de actos (o relaciones), una distinción según la cual el juicio, en términos propios, es la relación dual y la predicación es la relación múltiple. Si tomamos en cuenta la simbolización de los juicios y otras actitudes cognitivas propuesta por Russell, podríamos introducir esa distinción de la siguiente manera (donde ' $x$ ' es una variable cuyo valor, para cada ejemplar del tipo, es el agente correspondiente):

$\operatorname{Juzgar}(x,<$ Lara es gris $>)$,

Predicar(x, Lara, ser gris).

Como acabamos de ver, se trata de distintos tipos de actos (o relaciones):

$\operatorname{Juzgar}(x,<$ Lara es gris $>) \neq \operatorname{Predicar}(x$, Lara, ser gris $)$.

Esto no excluye, sin embargo, que haya una identidad entre un juicio y una predicación como ejemplares de ambos tipos:

un ejemplar de $\operatorname{Juzgar}(x,<$ Lara es gris $>)=$ un ejemplar de Predi$\operatorname{car}(x$, Lara, ser gris).

Supongamos que en una ocasión dada el escritor Coetzee juzga que Lara es gris. Dado que él puede realizar este tipo de acto cognitivo en 
otras ocasiones, resulta que su juicio es un ejemplar del tipo Juzgar (Coetzee, <Lara es gris $>$ ). Pero este tipo de acto es, a su vez, un sub-tipo de $\operatorname{Juzgar}(x,<$ Lara es gris $>$ ), en el sentido de que un ejemplar de Juzgar (Coetzee, <Lara es gris $>$ ) es también un ejemplar de Juzgar $(x,<$ Lara es gris $>$ ) sin que se dé la conversa. En efecto, siempre que Coetzee juzga que Lara es gris, un agente juzga que Lara es gris, pero no siempre que un agente juzga que Lara es gris, ese agente es Coetzee. Por esta razón, es $\operatorname{Juzgar}(x,<$ Lara es gris $>$ ), y no Juzgar(Coetzee, <Lara es gris $>$ ), el tipo de acto (o relación) que consiste en juzgar que Lara es gris. Por otra parte, tenemos que distinguir entre $\operatorname{Juzgar}(x,<$ Lara es gris $>$ ) y Predicar( $x$, Lara, ser gris). Pero esto no excluye que el acto particular que Coetzee lleva a cabo cuando juzga que Lara es gris sea también un ejemplar de Predicar(x, Lara, ser gris), lo que está en sintonía con una teoría cognitivista de las proposiciones basada en la predicación. Quizá sea mejor decir, por usar la terminología de Soames 2014b (pp. 228229), que el suceso mental en el que Coetzee juzga que Lara es gris es el mismo suceso en el que Coetzee predica ser gris de Lara. En un mismo suceso mental, Coetzee realizaría dos actos particulares distintos.

Sea como sea, está claro que, aunque se trate de distintos tipos de actos (o relaciones), una teoría cognitivista de las proposiciones debe explicar la actitud cognitiva básica, en este caso el juicio, en términos de predicación. A este respecto, Hanks sostiene, sin profundizar en la idea, que la relación entre dichos tipos de actos es una relación de fundación (grounding):

Un sujeto comporta la relación de juzgar a una proposición en virtud del hecho de que ha llevado a cabo un acto de predicación. El acto de predicación funda el juicio, pero la relación de predicación no es idéntica a la relación de juzgar. (Hanks 2015, p. 161; véase también pp. 46-47)

De acuerdo con la simbolización empleada en un caso ilustrativo, esa relación es:

$\operatorname{Predicar}(x$, Lara, ser gris) funda $\operatorname{Juzgar}(x,<$ Lara es gris $>$ ).

Como se sabe, hablar de fundación es hablar de una relación irreflexiva, asimétrica y transitiva que es, por un lado, distinta de la causalidad y no es, por otro lado, puramente modal. Cuando se dice que un hecho $[p]$ funda un hecho disyuntivo $[p o q]$, no nos referimos a una relación de causalidad entre ambos hechos, sino a una relación por la que un hecho determina o explica (no causalmente) otro hecho. Además, la fundación es una relación metafísica más fina que, por ejemplo, la ne- 
cesitación metafísica. Sin duda, todo mundo metafísicamente posible en el que $[p]$ es el caso es un mundo en el que [ $p$ o $q]$ es el caso. Pero también todo mundo metafísicamente posible en el que Coetzee escribió Verano es un mundo en el que $2+2=4$, sin que aquí un hecho se determine o explique por el otro hecho. Seguiré a Correia 2013 al considerar que la relación de fundación consiste en verdades esenciales acerca de una entidad fundada que la conectan con ciertas entidades que así resultan ser fundantes de ella. (Aunque para algunos autores sólo los hechos pueden ser los relata de dicha relación, supondré que también otras entidades, como los actos o relaciones, pueden desempeñar ese papel). Entonces, afirmar que Predicar( $x$, Lara, ser gris) funda $\operatorname{Juzgar}(x$, $<$ Lara es gris $>$ ) es afirmar que hay verdades esenciales acerca del acto de juzgar que Lara es gris que conectan este tipo de acto con el acto de predicar ser gris de Lara.

Por otra parte, en Hanks 2015 (pp. 161-163) y Hanks 2007 (pp. 137-141) se explica la distinción entre el juicio y la predicación en términos de la crítica de Wittgenstein a la teoría del juicio como relación múltiple. Según su interpretación de esta crítica, Wittgenstein partiría de la concepción fregeana según la cual juzgar es reconocer algo como verdadero para sostener que el juicio debe consistir en una relación con un sentido, es decir, una sola entidad unitaria que es así portadora de la verdad o la falsedad. Ésta es la razón por la que no sería posible juzgar un sinsentido. Pero resulta que una relación múltiple sería una relación con una colección de entidades (al menos un objeto y una propiedad) sin la unidad necesaria para que el acto de juzgar pudiera ser verdadero o falso. En la medida en que Hanks introduce la predicación como una relación múltiple, se sigue que, a diferencia del juicio como una relación dual con un sentido, el acto de predicar no puede ser verdadero o falso. ${ }^{4}$

Consideraré ahora las razones por las que, a mi modo de ver, las ideas expuestas no son satisfactorias. Téngase en cuenta, en primer lugar, que Hanks parece que reestablece el problema de la unidad de la proposición, o el problema de la representación tal y como se introdujo aquí, que supuestamente la teoría cognitivista había solucionado. En efecto, se trataba de que concebir las proposiciones como tipos de actos predicativos explicaría el hecho de que las proposiciones tengan propiedades representacionales y de que, por lo tanto, puedan ser ver-

\footnotetext{
${ }^{4}$ De modo que Hanks está hablando del juicio, no de la predicación misma, cuando dice cosas como esta: "Un acto de predicar una propiedad de un objeto es verdadero o falso en la medida en que [el objeto] satisfaga o deje de satisfacer las condiciones de corrección determinadas por la propiedad" (Hanks 2015, p. 66).
} 
daderas o falsas. Esto significa que, por ejemplo, el acto de predicar ser gris de Lara debiera tener la unidad necesaria para constituir una representación de Lara como gris, y en ese caso ser verdadero o falso. De otro modo, la proposición < Lara es gris> no podría ser, respecto a su carácter representacional, el tipo de acto que consiste en predicar ser gris de Lara. En consecuencia, Hanks, cuya propuesta no resolvería el problema de la representación proposicional en términos de predicación, tendrá que decir que la proposición < Lara es gris $>$ es, respecto a su carácter representacional, el tipo de acto consistente en juzgar esa proposición:

$$
<\text { Lara es gris }>=\operatorname{Juzgar}(x,<\text { Lara es gris }>) .^{5}
$$

Una segunda objeción a este resultado es que, como es obvio, la relación dual con una proposición que ya figura en el lado derecho de la igualdad como una entidad unitaria, capaz de ser verdadera o falsa, no puede explicar la unidad de esa misma proposición en el lado izquierdo de la igualdad. ${ }^{6}$ Veámoslo de esta otra manera. Es razonable suponer que el acto cognitivo invocado en el lado derecho de la igualdad correcta explicará la naturaleza de la proposición en un sentido en el que los demás actos (o actitudes) cognitivos hacia esa proposición no pueden hacerlo. Justo el hecho de que el acto cognitivo en cuestión tenga ese poder explicativo permitiría entender los demás actos (o actitudes) cognitivos como relaciones duales con proposiciones cuya naturaleza recibe una explicación independiente. Por eso, mientras que dicho acto cognitivo explicaría el carácter representacional de la proposición, la idea es que el orden de la explicación se invierte en el caso de los demás actos (o actitudes) cognitivos. Por ejemplo, el acto de suponer la proposición <Lara es gris $>$ es una representación de Lara como gris porque la proposición <Lara es gris $>$ es una representación de Lara como gris. No hay ningún problema cuando el acto cognitivo privilegiado es la predicación, pues no se invoca una relación dual cuyo carácter representacional dependa de la correspondiente proposición. Pero el juicio concebido como una relación dual no puede explicar la naturaleza de la proposición que ya figura en el lado derecho de la igualdad con el carácter representacional que justo necesitaba una explicación.

\footnotetext{
${ }^{5}$ No debe haber dudas sobre la corrección formal de esta oración: el predicado " $x=y$ " es un predicado de primer orden porque la expresión " $<$ Lara es gris $>$ " es un término para referirse a una proposición y la expresión "Juzgar $(x,<$ Lara es gris $>$ )" es un término para referirse a un tipo de acto (o relación).

${ }^{6}$ Speaks 2014 (pp. 159-160) ofrece una consideración en este mismo sentido.
} 
Más aún, surge ahora un problema con la propuesta de fundación entre Predicar( $x$, Lara, ser gris) como entidad fundante y $\operatorname{Juzgar}(x$, $<$ Lara es gris $>$ ) como entidad fundada. Como vimos, la fundación es una relación fina de la que se sigue una necesitación metafísica entre entidades: todo mundo metafísicamente posible en el que $[p]$ es el caso es un mundo en el que [ $p$ o $q]$ es el caso. Al menos eso es lo que ocurre si se trata de una fundación plena. Por el contrario, una fundación que sólo es parcial no conlleva necesitación metafísica. Una fundación parcial es, por ejemplo, la que parece darse entre un hecho $[p]$ y un hecho conjuntivo $[p \mathrm{y} q]$, y al menos para muchos hechos de esa forma, resulta que no todo mundo metafísicamente posible en el que $[p]$ es el caso es un mundo en el que también $[p$ y $q$ ] es el caso (Bliss y Trogdon 2016). Pero supongamos que, como sostiene Hanks, el acto de predicar ser gris de Lara no puede, a diferencia del juicio de que Lara es gris, ser verdadero o falso. No parece que, en tal caso, la predicación como entidad fundante pueda determinar o explicar por completo el juicio correspondiente como una entidad fundada. Por lo menos no podrá explicarse en términos de predicación la capacidad del juicio para ser verdadero o falso si el hecho de que la predicación es una relación con una colección de entidades no le permite tener la unidad necesaria para ser ella misma un acto verdadero o falso. De otro modo, estaríamos ante el resultado absurdo de que el acto de predicar ser gris de Lara no tiene la unidad necesaria para ser verdadero o falso y, sin embargo, sí tiene la unidad necesaria para hacer que el juicio de que Lara es gris pueda ser verdadero o falso (o, en términos equivalentes, que la proposición < Lara es gris > sea portadora de la verdad o la falsedad). ${ }^{7}$ Deberíamos concluir entonces que Hanks sólo tiene derecho a hablar de una fundación parcial:

$\operatorname{Predicar}(x$, Lara, ser gris) funda parcialmente $\operatorname{Juzgar}(x,<$ Lara es gris $>$ ).

Sin duda, la fundación parcial no excluye la necesitación metafísica: el hecho [Coetzee es escritor] sólo funda de manera parcial el hecho conjuntivo [Coetzee es escritor y $3+3=6$ ] y, sin embargo, todo mundo metafísicamente posible en el que [Coetzee es escritor] es el caso es un mundo en el que [Coetzee es escritor y $3+3=6$ ] es el

${ }^{7}$ Una vez más debe interpretarse que Hanks habla del juicio, y no de la predicación misma, cuando dice: “[...]los actos de predicación van a ser verdaderos o falsos, lo que tienen que ser para poder fundar las condiciones de verdad de las proposiciones" (Hanks 2015, p. 40). 
caso. ${ }^{8}$ Pero esta consideración no se puede usar para sostener que la relación entre Predicar( $x$, Lara, ser gris) y Juzgar $(x,<$ Lara es gris $>$ ) no sólo es de fundación parcial, sino también de necesitación metafísica. Téngase en cuenta primero que, al ser el hecho [Coetzee es escritor] uno de los constituyentes del hecho [Coetzee es escritor y $3+3=6$ ], la necesitación metafísica entre ambos es equivalente a la necesitación entre [Coetzee es escritor] y [3+3=6]. Y aquí la necesitación metafísica entre el hecho [Coetzee es escritor] y el hecho $[3+3=6]$ no se explica en términos de una fundación entre ellos, que no están en una relación de fundación de ningún tipo, sino que hay una explicación independiente en términos de que $[3+3=6]$ es el caso en todo mundo metafísicamente posible. Ahora bien, puesto que no parece haber ejemplares de $\operatorname{Juzgar}\left(x,<\right.$ Lara es gris $>$ ) en todo mundo metafísicamente posible ${ }^{9} \mathrm{ni}$ parece haber otra explicación independiente, ${ }^{10}$ se sigue que no puede darse una explicación independiente de la necesitación metafísica entre $\operatorname{Predicar}(x$, Lara, ser gris) y Juzgar $(x,<$ Lara es gris $>$ ). Dicha necesitación tendría entonces que explicarse en términos de la fundación entre $\operatorname{Predicar}(x$, Lara, $\operatorname{ser}$ gris) y Juzgar $(x,<$ Lara es gris $>$ ). Pero ya sabemos que no puede tratarse de fundación parcial, dado que la fundación parcial no conlleva una necesitación metafísica. Por lo tanto, debe tratarse de una fundación plena, que es justo la idea contra la que he argumentado antes.

Así, en la medida en que, por un lado, la relación entre $\operatorname{Predicar}(x$, Lara, ser gris) y Juzgar $(x,<$ Lara es gris $>$ ) es de fundación parcial y, por otro lado, no parece haber una explicación independiente de la necesitación metafísica entre ambos, es legítimo concluir:

No todo mundo metafísicamente posible en el que algún y es un ejemplar de Predicar( $x$, Lara, ser gris) es un mundo en el que $y$ es un ejemplar de $\operatorname{Juzgar}(x,<$ Lara es gris $>$ ).

${ }^{8}$ Debo esta consideración a un árbitro anónimo de la revista.

${ }^{9}$ Como es obvio, no hay ejemplares de ese tipo de juicio en los mundos metafísicamente posibles en los que no haya agentes que puedan realizar el juicio ni en los mundos metafísicamente posibles en los que dichos agentes existen pero no llegan a realizarlo.

${ }^{10}$ Debido a que tampoco parece haber una explicación independiente (es decir, independiente respecto a la fundación) que dé cuenta de que sólo haya ejemplares de Juzgar $(x,<$ Lara es gris $>$ ) en un conjunto restringido de mundos metafísicamente posibles $\left\{m_{\mathrm{j}}\right\}$ tal que ese conjunto es idéntico a, o al menos incluye, el conjunto de mundos metafísicamente posibles $\left\{m_{\mathrm{p}}\right\}$ en los que hay ejemplares de $\operatorname{Predicar}(x$, Lara, ser gris). 
Adviértase ahora que esto introduce un compromiso con actos de predicación que podrían no ir acompañados de su juicio correspondiente (ni, al ser el juicio la actitud cognitiva básica, de otra actitud cognitiva). Pero hay al menos dos consideraciones por las que este resultado no es aceptable. En primer lugar, es contrario a la tesis de Hanks en favor del juicio como actitud cognitiva básica, según la cual toda predicación conlleva una fuerza afirmativa o asertórica (aunque sólo sea prima facie). Esto es debido a que, en un mundo metafísicamente posible en el que un ejemplar de Predicar( $x$, Lara, ser gris) no fuese también primero un ejemplar de $\operatorname{Juzgar}(x,<$ Lara es gris $>$ ) incluso cuando hay cancelación de la fuerza, el acto de predicar ser gris de Lara no tendría inicialmente una fuerza afirmativa. Más relevante es el hecho de que dichos actos de predicación parecen misteriosos, dado que no se ve qué puede estar haciendo un agente que predica ser gris de Lara sin tener a la vez una u otra actitud cognitiva. ¿Qué sería una predicación que no se manifestara como un juicio o, por ejemplo, como una duda? No se entiende el papel que desempeñaría esa predicación en la vida cognitiva de un agente que al mismo tiempo no juzgara o concibiera o supusiera o negara (entre otros actos o actitudes posibles) que Lara es gris, cuando justo la predicación no se identificó en primer lugar como un acto que pudiera tener una "existencia separada". Es bastante incomprensible que, una vez que se establece un inventario de todas las actitudes cognitivas posibles, aún haya algo más que un agente pueda hacer con sólo llevar a cabo un acto de predicación.

\section{Una propuesta cognitivista con el juicio como relación múltiple}

A continuación propondré la idea de que juzgar es predicar, lo que significa que el juicio mismo, y no un acto predicativo distinto, es una relación múltiple. Así, por ejemplo, el juicio acerca del color gris de Lara será idéntico al acto de predicar ser gris de Lara:

Juzgar/Predicar(x, Lara, ser gris).

La predicación en que consiste este juicio explica tanto su carácter múltiple como su carácter unitario. En efecto, ese acto de juzgar/predicar relaciona a un agente con dos entidades de tal modo - a saber, predicando una propiedad de un objeto- que el acto mismo tiene la unidad necesaria para ser verdadero o falso. Se trata de que el agente usa la propiedad en cuestión como un predicado o, en otras palabras, que dicha propiedad comparece predicativamente en el juicio (Lebens 
2017, pp. 183-187; Sainsbury 2002, pp. 106-110). Así, la predicación garantiza que el juicio sea una entidad unitaria y no simplemente una colección de otras entidades. A este respecto puede compararse con la relación de instanciación en virtud de la cual Lara instancia la propiedad de ser gris. En este caso, la instanciación es una relación entre dos entidades que, sin embargo, compone una entidad unitaria, un estado de cosas, cuyos constituyentes son Lara y el color gris (Armstrong 1997, pp. 116-119). ${ }^{11}$ Puede decirse entonces que la predicación en el juicio es la contraparte de la instanciación en el estado de cosas, pues el acto de juzgar/predicar es verdadero si y sólo si Lara instancia el color gris; o, en términos equivalentes, ese acto es verdadero si y sólo si Lara y el color gris están unidos en un estado de cosas. También puede decirse, como afirma Lebens, que la propiedad en cuestión comparece de dos maneras diferentes: en el mundo se presenta como instanciada por el objeto en un estado de cosas, mientras que en el juicio se presenta como predicada del objeto por el agente sin que el objeto y la propiedad estén unidos en un estado de cosas por la relación de instanciación.

En una teoría cognitivista de las proposiciones, resulta ahora que una proposición es, de manera indistinta, un tipo de juicio o un tipo de acto predicativo:

$$
<\text { Lara es gris }>=\text { Juzgar/Predicar( } x \text {, Lara, ser gris) } .
$$

Se reestablece así la idea de que el acto cognitivo que figura en el lado derecho de la igualdad puede explicar o clarificar la naturaleza de la proposición que figura en el lado izquierdo. En particular, la proposición < Lara es gris > tiene la unidad necesaria para ser una representación de Lara como gris (y, por lo tanto, para ser portadora de la verdad o la falsedad) porque, como hemos visto, el acto de predicar ser gris de Lara tiene la unidad necesaria para ser una representación de Lara como gris (y, por lo tanto, para ser verdadero o falso).

Al margen de otras consideraciones -incluso más relevantespara seguir hablando de proposiciones, sugerí que un aspecto central de la teoría sería explicar el carácter representacional de las proposiciones en términos del carácter representacional de una actitud cognitiva básica, para luego explicar el carácter representacional de las demás actitudes cognitivas en términos del carácter representacional de las

\footnotetext{
${ }^{11}$ En realidad, para Armstrong hablar de instanciación es sólo un modo conveniente de hablar: los estados de cosas son primitivos respecto a los particulares y universales que los constituyen y, por lo tanto, la instanciación de un universal por un particular es sólo el estado de cosas mismo.
} 
proposiciones. En este sentido, las actitudes cognitivas que no son básicas deben entenderse como relaciones duales con proposiciones. Entre ellas se encuentran:

Concebir $(x,<$ Lara es gris $>$ ),

$\operatorname{Dudar}(x,<$ Lara es gris $>)$,

Suponer $(x,<$ Lara es gris $>)$,

$\operatorname{Negar}(x,<$ Lara es gris $>$ ), donde una negación es un rechazo (rejection).

Mi propósito entonces es mostrar cómo puede combinarse una teoría del juicio - la actitud cognitiva básica- como una relación múltiple con una teoría de las actitudes cognitivas en general como relaciones duales. La propuesta aquí es, claro está, que las actitudes cognitivas, como actitudes hacia las proposiciones, son en general actitudes hacia un tipo de juicio que el agente podría o no realizar. Por ejemplo, podemos decir que Concebir $(x,<$ Lara es gris $>$ ) es simplemente el tipo de acto que consiste en considerar, o tener presente en la mente, el tipo Juzgar/ Predicar( $x$, Lara, ser gris). Quizá Suponer $(x,<$ Lara es gris $>$ ) se distingue de ese tipo de acto en que es el acto que consiste en considerar el tipo Juzgar/Predicar( $x$, Lara, ser gris) para examinar cuáles serían las consecuencias, es decir, cuáles otros tipos de juicios se deberían realizar, si se realizara ese juicio o predicación. En el caso de $\operatorname{Dudar}(x,<$ Lara es gris $>$ ) se trataría del tipo de acto que consiste en suspender la realización del tipo Juzgar/Predicar(x, Lara, ser gris) como resultado de no encontrar evidencia suficiente a favor de ese juicio. A su vez, Negar( $x$, $<$ Lara es gris $>$ ) se distinguiría de ese tipo de acto en que es el acto que consiste en rechazar la realización del tipo Juzgar/Predicar( $x$, Lara, ser gris) como resultado de encontrar evidencia suficiente en contra de ese juicio. ${ }^{12}$

Téngase en cuenta que estos actos cognitivos que no son juicios no son, por la misma razón, actos que consistan en predicar: Concebir

${ }^{12}$ Este tipo de acto no debe confundirse con el juicio que se obtiene de negar primero la propiedad de ser gris y entonces predicar no-ser gris de Lara: Juzgar/ Predicar( $x$, Lara, no-ser gris). Ofrecer un análisis preciso de las distintas actitudes cognitivas excede los límites de este artículo; su caracterización ya es de por sí objeto de discusión con independencia de que se adopte o no una teoría cognitivista de las proposiciones. 
$(x,<$ Lara es gris $>$ ) no es predicar ser gris de Lara ni es ninguna otra predicación, sino que es considerar, o tener presente en la mente, esa predicación como un tipo de juicio que el agente podría realizar. En otras palabras, Concebir $(x,<$ Lara es gris $>$ ) es el acto de considerar cierto acto de predicación, Juzgar/Predicar( $x$, Lara, ser gris), sin llevarlo a cabo. De la misma forma, esto significa que un ejemplar de Concebir $(x,<$ Lara es gris $>$ ) no es un ejemplar de la proposición < Lara es gris $>$ como el tipo de acto que consiste en predicar ser gris de Lara. Puede objetarse ahora que, puesto que la teoría cognitivista pretendía explicar la representación proposicional en términos de la predicación, deberíamos concluir de modo absurdo que concebir una proposición no es representarse las cosas de tal o cual manera. Pero esta objeción no se hace cargo de que el término "representación proposicional" tiene dos sentidos diferentes. En primer lugar, hablamos de representación proposicional cuando nos referimos al carácter representacional de las proposiciones y, en este sentido, la idea es precisamente que las proposiciones tienen propiedades representacionales porque son tipos de juicios y, por lo tanto, son tipos de actos predicativos. A continuación, podemos explicar la representación proposicional de la que hablamos cuando nos referimos a los actos (o actitudes) cognitivos como relaciones duales con proposiciones en términos de la representación proposicional en el primer sentido. Un acto como Concebir ( $x$, <Lara es gris $>$ ) es una representación de Lara como gris porque, aunque no es el tipo de acto que consiste en predicar ser gris de Lara, toma su carácter representacional de la proposición $<$ Lara es gris $>$ como el tipo de acto que consiste en predicar ser gris de Lara. Sin duda, esto implica que puede haber una representación proposicional sin que haya un acto de predicación. Sin embargo, la representación proposicional, en el segundo sentido, no deja de explicarse en términos de la predicación.

Una de las ventajas de la propuesta que presento es que puede justificar la distinción entre concebir y juzgar en relación con la fuerza afirmativa o asertórica sin introducir, como Hanks, una operación de cancelación. Adviértase que, dado que no hay una distinción entre juzgar y predicar, resulta que la predicación es de naturaleza afirmativa. Además, en la medida en que la predicación es la noción primitiva, parece que la predicación misma debe ser responsable de la fuerza afirmativa del juicio. Se sigue así que los actos (o actitudes) cognitivos que no son actos de predicación no tienen tampoco fuerza afirmativa (ni siquiera prima facie, como sostiene Hanks). Por ejemplo, Concebir( $x$, $<$ Lara es gris $>$ ) será un tipo de acto que carece de cualquier fuerza afirmativa. Piénsese ahora en la proposición disyuntiva < Lara es gris o 
Luna es blanca>. Esta proposición puede verse como el tipo de juicio que consiste en predicar la relación de disyunción de las proposiciones $<$ Lara es gris $>$ y $<$ Luna es blanca $>$ :

Juzgar/Predicar $(x,<$ Lara es gris $>$, < Luna es blanca $>$, ser disyuntos).

Como está claro, también las proposiciones $<$ Lara es gris $>\mathrm{y}<$ Luna es blanca $>$ son tipos de juicios, es decir, tipos de actos predicativos. Pues bien, la idea es que, cuando un agente realiza un juicio disyuntivo de este tipo, predica la disyunción de las proposiciones < Lara es gris $>$ y $<$ Luna es blanca $>$ sin realizar juicios de estos mismos tipos. Esto significa que el agente no predica ser gris de Lara ni predica ser blanca de Luna. Podría explicarse de este modo que las proposiciones < Lara es gris $>$ y $<$ Luna es blanca $>$ aparezcan como proposiciones no aseveradas cuando el agente asevera la proposición < Lara es gris o Luna es blanca $>$, como suele decirse. (Por supuesto, hablar aquí de "aseverar una proposición" es un giro en el lenguaje proposicional habitual que, sin embargo, no debe llevarnos a interpretar la aserción como una relación dual). Más aún, el hecho de que las proposiciones $<$ Lara es gris $>$ y $<$ Luna es blanca $>$ aparezcan como proposiciones no aseveradas puede interpretarse como el hecho de que el agente sólo debe concebir dichas proposiciones, pues precisamente concebir una proposición no implica aseverarla (es decir, no implica llevar a cabo el correspondiente acto de predicación):

Concebir $(x,<$ Lara es gris $>)$,

Concebir $(x,<$ Luna es blanca $>)$.

Más bien, mediante el acto de concebir dichas proposiciones el agente las convierte en objetos para predicar después su disyunción. Propongo entonces que, para realizar el juicio que consiste en predicar la disyunción de las proposiciones < Lara es gris $>$ y $<$ Luna es blanca $>$, un agente debe primero concebir estas proposiciones (sin aseverarlas) para así convertirlas en objetos de la predicación.

Este análisis de la distinción entre concebir y juzgar es superior al de Hanks en varios aspectos. Como vimos, conserva tanto la idea de que el juicio (y la aserción) es la actitud cognitiva básica como la idea de que la predicación es de naturaleza afirmativa; sin embargo, al mismo tiempo evita extender la fuerza afirmativa, dado que otros actos (o actitudes) cognitivos no son actos de predicación. Tal es la razón por la que 
no es necesario introducir una operación de cancelación que transforme dichos actos cognitivos, como actos de predicación con fuerza afirmativa, en actos sin fuerza afirmativa. Recordemos, por otro lado, que, según la propuesta de Hanks, un contexto de cancelación es tal que el agente debe llevar a cabo un acto de predicación, es decir, producir un ejemplar de cierta proposición no aseverada (como resultado de la cancelación), para convertir esa proposición, mediante una operación de cambio de objetivo, en objeto de una predicación ulterior. Por ejemplo, un agente empezará predicando ser gris de Lara de tal modo que, cancelada la fuerza afirmativa, el acto ulterior de predicar la disyunción se dirija a la proposición <Lara es gris $>$ como objeto de predicación en el juicio disyuntivo. Puede cuestionarse de entrada la necesidad del cambio de objetivo si, como sostiene Hanks, cualquier acto de predicación conlleva también una relación dual con la correspondiente proposición. En efecto, supongamos que aquí el acto de predicar ser gris de Lara conlleva también el acto de concebir la proposición < Lara es gris > (como resultado de la cancelación). Puesto que este acto de concebir es una relación dual, pone la proposición <Lara es gris $>$ a disposición del agente para poder predicar de ella la disyunción y, por lo tanto, no es necesario un cambio de objetivo que consista en que el acto de predicar ser gris de Lara convierta esa proposición en objeto de predicación. Pero esto muestra a la vez que el acto de predicar ser gris de Lara es una pieza inútil en el engranaje. Justo mi propuesta, según la cual el acto de concebir una proposición no es ni involucra un acto de predicación, establece que el agente sólo debe concebir la proposición < Lara es gris $>$ para convertirla en un objeto de predicación. Puede verse ahora que esta propuesta es mucho más económica: en la medida en que concebir no consiste en predicar, resulta que concebir una proposición no requiere cancelar la fuerza afirmativa que es propia de la predicación ni requiere cambiar el objetivo de la predicación para poder realizar un juicio que tiene esa proposición como objeto.

Podría plantearse una objeción a la idea del juicio como una actitud cognitiva básica. ${ }^{13}$ En la primera sección vimos que, tanto para Soames como para Hanks, la actitud básica involucra cierto tipo de acto cognitivo al que las demás actitudes añaden, además, algún otro rasgo. En ambos casos ese acto es el acto de predicación, y Hanks sostiene que el rasgo adicional es la cancelación de la fuerza afirmativa del juicio. Pero téngase en cuenta que, como argumentan varios autores (Conant 2020, pp. 825-826; Bronzo 2021, pp. 3112-3122; Reiland 2013,

${ }^{13}$ Debo esta consideración a un árbitro anónimo de la revista. 
pp. 242-243), la propuesta de Hanks podría acabar en una concepción conjuntivista del juicio como la de Soames. Puesto que, por ejemplo, concebir una proposición sería llevar a cabo un acto de predicación sin fuerza afirmativa, la cancelación parece consistir en sustraer dicha fuerza al juicio. Es decir, la cancelación de la fuerza se relacionaría con el juicio como la sustracción se relaciona con la adición. No obstante, esto significa que el juicio está compuesto de predicación y afirmación (o de contenido y fuerza). En este sentido, Soames tendría razón cuando afirma que el acto básico, o simple, es concebir una proposición. Sin embargo, la concepción del juicio que he presentado aquí no es conjuntivista, dado que no cabe sustraer la fuerza afirmativa al juicio y obtener así algún acto (o actitud) cognitivo que sólo consista en predicar. No hay actos de predicación que no sean juicios. Por lo tanto, mi propuesta justifica la idea del juicio como un acto simple, que no se compone de predicación y afirmación. Además, no genera el problema de explicar en qué sentido concebir una proposición es llevar a cabo un acto de predicación sin fuerza afirmativa, si el juicio es un acto simple del que no se puede sustraer dicha fuerza. Recordemos una vez más que concebir una proposición no es llevar a cabo un acto de predicación cuya fuerza afirmativa tuviera entonces que cancelarse.

Mi propuesta tiene semejanzas con algunas ideas recientes de Reiland 2019. Hablando en los términos de Frege de captar (grasping) una proposición cuando hablamos del acto de concebir, Reiland salvaguarda la naturaleza afirmativa de la predicación cuando sostiene que captar una proposición no requiere, a diferencia de las demás actitudes cognitivas, llevar a cabo el correspondiente acto predicativo. Para justificar esta idea, introduce la distinción entre ser el objeto y ser el contenido de una actitud cognitiva. Los juicios - y otras actitudes cognitivas- son actitudes que tienen como contenido una proposición en el sentido de que son actitudes sensibles a la verdad de esa proposición: si la proposición que se juzga es verdadera, entonces las cosas en el mundo son tal y como se juzga que son. Por el contrario, el acto de captar una proposición es un acto objetual en el sentido de que, como el acto de referirse a un objeto, no tiene un contenido que lo haga ser sensible a la verdad. Justo la proposición comparece como el objeto, no como el contenido, de ese acto (Reiland 2019, pp. 150-152). ${ }^{14}$ Parece entonces que, así

\footnotetext{
${ }^{14}$ En esto también podría estar de acuerdo con Reiland, y entender así el acto de concebir una proposición como un acto objetual. Adviértase que también Frege pensaba algo parecido sobre captar una proposición (Hanks 2015, p. 40), a la vez que no veía problema con que la proposición que comparece como objeto (por
} 
como el acto de captar no requiere llevar a cabo ninguna predicación porque sólo tiene una proposición como objeto, el juicio sí requiere llevar a cabo un acto de predicación porque tiene una proposición como contenido. Pero esta propuesta parece invertir el orden de la explicación según el cual, como diría Hanks, es el hecho de que un juicio involucre un acto de predicación lo que debería explicar que el juicio tenga un contenido y de esta forma sea sensible a la verdad de ese contenido. Al margen de esta crítica, podemos ver que Reiland sigue considerando el juicio como una relación dual, no como una relación múltiple (aunque los juicios también sean o involucren actos de predicación).

Otro planteamiento con algunas ideas semejantes es el de Michael Schmitz (en preparación). En principio, Schmitz es un crítico de Hanks al establecer una distinción entre contenido y fuerza que equivaldría a la distinción entre la predicación - que sólo articula la representación de un estado de cosas- y el juicio o la aserción —que es la adopción de un compromiso con la realidad de ese estado de cosas-. Sin embargo, está del lado de Hanks cuando sostiene que una proposición, o su equivalente no proposicional, ${ }^{15}$ incorpora tanto la representación de un estado de cosas como la posición teórica hacia el mundo, en sus palabras, que es propia del compromiso. En efecto, un acto (o actitud) como concebir una proposición también sería una posición teórica que precisamente conlleva suspender el compromiso incorporado en la proposición. La proposición misma debe incorporar la posición teórica que es propia del compromiso y no sólo el contenido representacional porque, según Schmitz, no resulta inteligible suspender (ni, por ejemplo, cuestionar o simular) una posición teórica hacia el mundo sin saber cuál es esa posición teórica, lo que implica que dicha posición esté presente de algún modo. En este sentido, los actos (o actitudes) cognitivos distintos del juicio, por decirlo en mis términos, son actos de niveles más altos que los correspondientes juicios, pues se trata de posiciones teóricas hacia la posición teórica incorporada en esos juicios. Las semejanzas con mi propuesta pueden verse, primero, en la idea de que, al ser tipos de juicios, las proposiciones incorporan la fuerza afirmativa que es propia

ejemplo, en un juicio disyuntivo) conservara sus propiedades semánticas. Aunque el acto de captar/concebir no es verdadero ni falso, la proposición concebida mediante ese acto sí es verdadera o falsa; más aún, contribuye al juicio con las mismas condiciones de verdad (y, por lo tanto, con el mismo valor de verdad).

${ }^{15}$ En realidad, Schmitz no introduce proposiciones en su ontología, sino que pretende dar una explicación que sólo haga uso de actos de habla y actos intencionales. Por una cuestión de simplicidad, he preferido exponer su propuesta como si trabajara con proposiciones. 
de un juicio. Por ejemplo, la proposición <Lara es gris $>$ toma la fuerza afirmativa que es propia del juicio que consiste en predicar ser gris de Lara. Pero, en la medida en que, según mi propuesta, concebir esa proposición no es llevar a cabo esa predicación, la fuerza incorporada en la proposición se suspende. Además, las actitudes cognitivas distintas del juicio son actitudes hacia un juicio (una actitud hacia el mundo) que el agente podría adoptar y, en esta medida, son actitudes de niveles más altos. Sin embargo, la propuesta de Schmitz es también muy diferente; entre otras cosas, porque, por un lado, para él todos los actos (actitudes) cognitivos involucran una predicación y, por otro lado, no entiende el juicio (o aserción) como una relación múltiple.

Terminaré esta sección argumentando que el juicio no sólo tiene prioridad en la explicación de la representación proposicional, sino que, además, tiene prioridad en el desarrollo evolutivo de las otras actitudes cognitivas. Considérese el modo como un agente puede identificar, o estar familiarizado con, una proposición. Dado que tenemos conciencia-de-acción de nuestros actos tanto físicos como mentales (Peacocke 2008, cap. 5) en el sentido de ser conscientes de estar llevándolos a cabo, es razonable sostener que la conciencia-de-acción mediante la cual un agente es consciente de realizar un juicio es el modo más básico de identificar la correspondiente proposición. Quizá haya alguna modalidad no conceptual de conciencia-de-acción mediante la cual un agente puede ser consciente de su acto, como levantar un brazo, sin ser consciente del tipo de acto que está realizando, pero no parece que eso pueda ocurrir en el caso de los juicios, cuya naturaleza es conceptual. Parece entonces que, cuando realizo el juicio que consiste en predicar ser gris de Lara, debo ser consciente del tipo de acto predicativo que llevo a cabo, lo que significa que identifico de un modo práctico, por así decirlo, la proposición <Lara es gris $>$. Como era de esperarse, el ejercicio de la capacidad de juzgar no requiere la identificación previa de la correspondiente proposición sino que, por el contrario, esa identificación se produce por el ejercicio mismo de esa capacidad. Pero el asunto es diferente para el resto de las actitudes cognitivas. En la medida en que, por ejemplo, negar la proposición < Lara es gris > sólo es estar en una relación dual con esa proposición, resulta que el agente debe primero identificar la proposición para luego negarla. Podría decirse entonces que en estos casos identificamos la correspondiente proposición mediante una conciencia reflexiva de los actos cognitivos, y en especial los actos de predicación, que hemos llevado a cabo. Se trataría de la conciencia mediante la cual convierto los actos cognitivos realizados en objetos de una atención, o cognición, explícita (Soames 2014a, pp. 98, 
104; Soames 2015, pp. 20-21). Puedo, por ejemplo, ser consciente reflexivamente de haber predicado ser gris de Lara, o de haber dudado de que Lara sea gris, con lo que identifico la proposición < Lara es gris> para negarla a continuación. ${ }^{16}$

Pues bien, estas consideraciones sugieren que la capacidad de juzgar, o al menos su ejercicio, precede en términos evolutivos a la capacidad de tener otras actitudes cognitivas, en el sentido de que podría haber un organismo que ejerciese ya la capacidad de juzgar sin ser capaz todavía de negar una proposición (Reiland 2019, p. 150). Un organismo en este estado, ya sea en el nivel ontogenético o en el filogenético, sería capaz de predicar ser gris de Lara y, por lo tanto, tendría una conciencia-de-acción del tipo de juicio que realiza sin haber desarrollado aún la conciencia reflexiva necesaria para poder negar la proposición $<$ Lara es gris $>$. Sin duda, dicho organismo habría identificado de un modo práctico la proposición < Lara es gris $>$, pero no estaría en condiciones de identificarla de un modo reflexivo para luego negarla. Adviértase por último que ese organismo sólo podría realizar juicios atómicos porque hacer, por ejemplo, un juicio que predica la disyunción de las proposiciones < Lara es gris $>$ y < Luna es blanca $>$ supone convertir esas proposiciones en objetos de la predicación, lo que precisa tener una conciencia reflexiva para así identificarlas primero y luego concebirlas en el juicio (Reiland 2019, pp. 154-158). ${ }^{17}$

\section{Los reportes del juicio como relación múltiple}

Una teoría del juicio como relación múltiple se enfrenta al desafío mayor de explicar los reportes mediante los cuales un agente atribuye un juicio a otro agente (o a sí mismo). ${ }^{18}$ La razón para ello es que la forma lógica de tales reportes parece sugerir que el juicio es una relación dual.

${ }^{16}$ También puede ser que identifiquemos esa proposición mediante una conciencia reflexiva de las semejanzas y diferencias entre actos cognitivos previos, como el acto de predicar ser gris de algún objeto a que no es Lara y el acto de predicar de Lara alguna propiedad $F$ que no es el color gris.

${ }^{17}$ Reiland no piensa en términos de una conciencia reflexiva. Sostiene que captar una proposición es un modo práctico de identificarla. Sin embargo, no se trata de identificar prácticamente una proposición porque se realizó el juicio correspondiente, sino de disponer de un modo práctico de presentación de esa proposición sólo como resultado de poseer la capacidad de juzgarla (Reiland 2019, pp. 152-154).

${ }^{18}$ A este desafío han respondido distintos autores como Jubien 2001 y Moltmann 2003. 
En efecto, supongamos que cierto agente realiza un reporte con la oración:

“Coetzee juzgó que Lara es gris".

Un análisis estándar de este reporte sostiene que la cláusula-que subordinada es un término singular que se refiere a la proposición <Lara es gris $>$ y que, por lo tanto, el reporte tiene la forma lógica de una expresión relacional con dos lugares para términos singulares: $x$ juzga $y$. Desde luego que se han propuesto muchos análisis alternativos, como la teoría según la cual la cláusula-que no es un término singular, sino una frase en la que la partícula "que" tiene un carácter sincategoremático cuya función es permitir que la cláusula-que exprese la misma proposición que la oración independiente "Lara es gris". Según esto, el reporte tiene una forma lógica híbrida entre una expresión predicativa con lugar para un término singular a la izquierda y una conectiva oracional con lugar para una oración a la derecha: $x$ juzga $p$ (Trueman 2021, pp. 159-168). ${ }^{19}$ Ahora bien, puesto que existe cierta independencia entre el análisis de los reportes que atribuyen un juicio y la concepción misma del juicio como una actitud cognitiva (Moltmann 2003, p. 108), nada impide que tanto uno como otro análisis se complemente con una concepción según la cual el juicio es una relación dual. El juicio reportado sería una relación dual entre Coetzee y la proposición < Lara es gris $>$, ya sea que la cláusula-que se refiera a esa proposición o, por el contrario, que constituya un modo de expresarla.

A continuación, simplemente daré por supuesto que el análisis estándar es el análisis correcto. En cualquier caso, la independencia que mencioné antes me permitirá mostrar que el análisis estándar es compatible con una concepción según la cual el juicio es una relación múltiple. Recuérdese, en primer lugar, que mi propuesta sobre el juicio como una relación múltiple forma parte de una teoría cognitivista de las proposiciones. Así, no se trata sólo de que un juicio sea un acto de predicación y, por lo tanto, una relación múltiple, sino también de que es una relación de ejemplificación con una proposición como un tipo de acto predicativo (o un tipo de juicio). Esto significa que, aunque no es un acto que toma una proposición como su objeto intencional, aún consideramos el juicio como un acto que consiste en cierta relación

\footnotetext{
${ }^{19}$ Una versión anterior de este análisis (Prior 1971, cap. 2) niega incluso que haya una cláusula-que como unidad sintáctica porque la partícula "que" pertenece al predicado, y así la expresión predicativa es de la forma: $x$ juzga-que $p$.
} 
con una proposición. A este respecto, sostendré que el juicio reportado por una emisión de "Coetzee juzgó que Lara es gris" es una relación de ejemplificación entre Coetzee y la proposición <Lara es gris $>$. Desde luego, en un sentido la relación de ejemplificación sólo se da entre esa proposición y el acto de predicación que Coetzee lleva a cabo, pues es justo dicho acto el ejemplar de la proposición. Pero Coetzee mismo está en una relación de ejemplificación con la proposición < Lara es gris $>$ en el sentido de que está en la relación de producir un ejemplar con esa proposición (y a partir de ahora hablaré de ejemplificación en este sentido). En consecuencia, la proposición que expresa el reporte anterior es:

$<$ Coetzee realizó un juicio del tipo < Lara es gris $>>$,

$<$ Coetzee produjo un ejemplar del tipo $<$ Lara es gris $>>{ }^{20}$

Supongamos entonces que, como establece el análisis estándar, la forma lógica del reporte es: $x$ juzga $y$. Pues bien, las emisiones de esta forma no se interpretarán ahora como si reportaran una relación dual entre un agente y la proposición (como objeto) a la que se refiere la cláusula-que que ocupa el lugar y. Se interpretarán como reportes de una relación de ejemplificación entre un agente y la proposición (como tipo) a la que se refiere la cláusula-que que ocupa el lugar $y$. Por supuesto, la relación de ejemplificación entre un agente y una proposición también es una relación dual, y ésa es la razón por la que el análisis estándar de los reportes que atribuyen juicios puede prestarse a una interpretación como ésta. Pero no se trata del juicio como una relación dual que consista en tener cierta actitud cognitiva hacia una proposición como su objeto intencional.

Para ser más precisos, considérese ahora la proposición $<$ Coetzee produjo un ejemplar del tipo < Lara es gris $>>$ como un tipo de juicio. En efecto, el agente que realiza el reporte con la oración "Coetzee juzgó que Lara es gris" realiza una aserción que, como un juicio particular, es un ejemplar de ese tipo proposicional. Según la teoría cognitivista de las proposiciones, resulta que esa proposición es el siguiente tipo de juicio:

${ }^{20} \mathrm{O}$, en términos equivalentes:

$<$ Coetzee realizó un juicio del tipo Juzgar/Predicar( $x$, Lara, ser gris) >,

$<$ Coetzee produjo un ejemplar del tipo Juzgar/Predicar(x, Lara, ser gris) $>$. 
Juzgar/Predicar( $x$, Coetzee, <Lara es gris $>$, producir un ejemplar). ${ }^{21}$

Como está claro, este juicio es el tipo de acto que consiste en predicar producir un ejemplar, o la relación de ejemplificación, de Coetzee y la proposición <Lara es gris $>$. Se sigue que el agente que hace el reporte tiene que convertir esa proposición en un objeto de predicación. Identificará la proposición < Lara es gris > mediante cierta conciencia reflexiva de, por ejemplo, las semejanzas y diferencias entre los actos cognitivos previos sobre la gata Lara, por una parte, y sobre la propiedad de ser gris, por otra parte, para luego concebirla en el reporte que predica producir un ejemplar de Coetzee y esa proposición. Sea esto como sea, vemos que la propuesta no impone al agente que realiza el reporte el requerimiento de predicar ser gris de Lara, como sostiene Hanks. Ciertamente, el agente debe tener alguna actitud cognitiva hacia la proposición < Lara es gris > para convertirla en un objeto de predicación, quizá sólo debe concebirla, pero ya sabemos que cualquier actitud cognitiva distinta del juicio, como concebir esa proposición, no conlleva predicar ser gris de Lara.

Ahora bien, mi propuesta no sostiene que si el reporte con la oración "Coetzee juzgó que Lara es gris" es verdadero, entonces el agente reportado, en este caso Coetzee, debe haber concebido la proposición $<$ Lara es gris $>$ o tener alguna otra actitud cognitiva hacia esa proposición. Recordemos que, puesto que una proposición sólo es un tipo de acto predicativo, producir un ejemplar de esa proposición es simplemente predicar ser gris de Lara. Ésta es, desde luego, la razón por la que el juicio como un acto de predicación puede ser tanto una relación múltiple como una relación dual en el sentido relevante aquí. Por ello, las condiciones de verdad de la proposición $<$ Coetzee produjo un ejemplar del tipo $<$ Lara es gris $>>$, o $<$ Coetzee realizó un juicio del tipo $<$ Lara es gris $>>$, pueden formularse indistintamente así:

La proposición <Coetzee produjo un ejemplar del tipo < Lara es gris $>>$ es verdadera si y sólo si Coetzee produjo un ejemplar del tipo $<$ Lara es gris $>$.

${ }^{21} \mathrm{O}$, en términos equivalentes:

Juzgar/Predicar(x, Coetzee, Juzgar/Predicar(y, Lara, ser gris), producir un ejemplar). 
La proposición <Coetzee produjo un ejemplar del tipo <Lara es gris $>>$ es verdadera si y sólo si Coetzee predicó ser gris de Lara.

Sin duda, el lado derecho del primer bicondicional menciona la proposición < Lara es gris> al especificar el tipo de acto cognitivo que Coetzee debió realizar para que la proposición $<$ Coetzee produjo un ejemplar del tipo <Lara es gris $>>$ sea verdadera. Pero dicha proposición no comparece como el objeto intencional del acto cognitivo que Coetzee realizó. Justo por ello el lado derecho del segundo bicondicional no menciona ya la proposición <Lara es gris $>$ ni, por lo tanto, ningún acto (o actitud) cognitivo hacia esa proposición. Dado entonces que, como cualquier emisión asertórica de una oración, un reporte tiene las mismas condiciones de verdad que la proposición que expresa, obtenemos también el siguiente bicondicional:

El reporte con la oración "Coetzee juzgó que Lara es gris" es verdadero si y sólo si Coetzee predicó ser gris de Lara.

Desde luego que podría plantearse la posibilidad de que el agente que hace el reporte tampoco hubiera concebido la proposición <Lara es gris $>$. En efecto, supongamos que realizara más bien un reporte con la oración:

"Coetzee predicó ser gris de Lara".

En este caso, la proposición que expresa el reporte es ahora:

$<$ Coetzee predicó ser gris de Lara $>$.

Y, según la teoría cognitivista de las proposiciones, esa proposición es el siguiente tipo de juicio:

Juzgar/Predicar(x, Coetzee, Lara, ser gris, predicar).

Por lo tanto, es el tipo de acto que consiste en predicar predicar de Coetzee, la gata Lara y la propiedad de ser gris. Como puede verse, este tipo de acto no toma la proposición < Lara es gris $>$ como objeto de predicación, pero el agente que realiza un reporte con la oración "Coetzee predicó ser gris de Lara" realiza precisamente una aserción que es un ejemplar de ese tipo. Luego, el agente no tiene primero que identificar la proposición < Lara es gris > para concebirla a continuación y así convertirla en objeto de predicación en el reporte. Adviértase que las proposiciones < Coetzee produjo un ejemplar del tipo < Lara es gris $>>$, 
o < Coetzee realizó un juicio del tipo <Lara es gris $>>$, y $<$ Coetzee predicó ser gris de Lara > tienen las mismas condiciones de verdad: ambas son verdaderas si y sólo si Coetzee predicó ser gris de Lara. Sin embargo, para una teoría cognitivista según la cual las proposiciones son tipos de juicios o tipos de actos predicativos, se trata en realidad de proposiciones distintas porque imponen distintos requerimientos al agente que realiza el reporte: en un caso, pero no en el otro, el agente debe concebir la proposición <Lara es gris > . A este respecto podríamos decir, siguiendo a Soames 2014a (pp. 105-106) y a Soames 2015 (pp. 39-41), que las proposiciones <Coetzee produjo un ejemplar del tipo <Lara es gris $>>$ y <Coetzee predicó ser gris de Lara $>$ son representacionalmente idénticas pero cognitivamente distintas.

Resulta legítimo preguntar por qué, en general, un agente tiene una disposición a realizar un reporte con la oración "Coetzee juzgó que Lara es gris" y no con la oración "Coetzee predicó ser gris de Lara", cuando la carga cognitiva parece ser mayor en el primer caso, al menos en el sentido de que realizar el primer reporte, pero no el segundo, requiere que dicho agente conciba la proposición <Lara es gris $>$. Este hecho puede explicarse en términos de la uniformidad lingüística que uno esperaría encontrar en los reportes de las actitudes cognitivas. Si suponemos que el lenguaje es una capacidad que sólo tienen los organismos que ya son capaces de identificar y concebir proposiciones, entonces tales organismos lingüísticos estarán en condiciones de realizar tanto el reporte de un juicio como el reporte, por ejemplo, de una duda o una negación. Puesto que una duda y una negación son actitudes cognitivas hacia proposiciones, es de esperarse que las expresiones lingüísticas para reportar tales actitudes sean construcciones con cláusulas-que que se refieren a las proposiciones correspondientes. Y una vez que el lenguaje incorpora este tipo de construcciones para reportar las distintas actitudes cognitivas, es una consecuencia de la uniformidad lingüística que un agente también esté dispuesto a reportar un juicio usando una construcción con una cláusula-que.

Consideremos entonces, para finalizar, el reporte de una actitud cognitiva distinta del juicio. Digamos que un agente realizó un reporte con la oración:

"Coetzee negó que Lara es gris".

Según la propuesta cognitivista desarrollada aquí, ese reporte expresa la siguiente proposición:

$<$ Coetzee rechazó realizar un juicio del tipo < Lara es gris $>>$, 
$<$ Coetzee rechazó producir un ejemplar del tipo <Lara es gris $>>$.

Como está claro, esta proposición es un tipo de juicio. En efecto, el agente que realiza un reporte con la oración "Coetzee negó que Lara es gris" realiza una aserción que es un ejemplar del siguiente tipo:

Juzgar/Predicar(x, Coetzee, <Lara es gris>, rechazar producir un ejemplar).

En la medida en que este juicio es el tipo de acto que consiste en predicar rechazar producir un ejemplar de Coetzee y la proposición <Lara es gris $>$, se sigue que el agente que reporta el juicio debe identificar y concebir esa proposición para convertirla en objeto de predicación.

Vemos entonces que el reporte con la oración "Coetzee negó que Lara es gris" debe interpretarse como el reporte de una relación dual entre Coetzee y la proposición <Lara es gris>. Luego, no es extraño que, como establece el análisis estándar, ese reporte tenga la forma lógica: $x$ niega $y$. Quiero mostrar ahora que rechazar producir un ejemplar de una proposición es en efecto negarla en el sentido de estar en una relación dual con esa proposición (como objeto). Téngase en cuenta, primero, que rechazar producir un ejemplar de la proposición < Lara es gris $>$ no es un tipo de juicio ni, por lo tanto, de predicación. Para serlo, tendría que ocurrir que un agente que rechazara producir un ejemplar de esa proposición estuviese produciendo un ejemplar de alguna otra proposición. Pero rechazar llevar a cabo cierto acto de predicación no consiste en llevar a cabo algún otro acto de predicación. ${ }^{22}$ Se sigue entonces que un agente que rechaza producir un ejemplar de la proposición <Lara es gris> no está en una relación de ejemplificación con alguna otra proposición. Por el contrario, dicho agente debe identificar reflexivamente la proposición < Lara es gris> para, a continuación, tomar la actitud cognitiva hacia esa proposición que consiste en rechazar producir un ejemplar de ella.

Por esta razón, la mención de la proposición <Lara es gris $>$ en las condiciones de verdad de la proposición $<$ Coetzee rechazó producir un ejemplar del tipo < Lara es gris $>>$ es ineliminable:

La proposición <Coetzee rechazó producir un ejemplar del tipo $<$ Lara es gris $>>$ es verdadera si y sólo si Coetzee rechazó producir un ejemplar del tipo <Lara es gris $>$.

\footnotetext{
${ }^{22}$ En este caso, eso sería posiblemente el resultado de confundir el tipo de acto $\operatorname{Negar}(x,<$ Lara es gris $>$ ) con el tipo de acto Juzgar/Predicar( $x$, Lara, no-ser gris).
} 
No sólo el lado derecho del bicondicional menciona la proposición $<$ Lara es gris $>$ al especificar el tipo de acto cognitivo que Coetzee debió realizar para que la proposición $<$ Coetzee rechazó producir un ejemplar del tipo <Lara es gris $>>$ sea verdadera. Además, dicha proposición comparece como el objeto intencional del acto cognitivo que Coetzee realizó después de identificarla mediante un ejercicio de la conciencia reflexiva. ${ }^{23}$ *

\section{REFERENCIAS BIBLIOGRÁFICAS}

Armstrong, David Malet, 1997, A World of States of Affairs, Cambridge University Press, Cambridge, <https://doi.org/10.1017/cbo9780511583308>.

Bronzo, Silver, 2021, "Propositional Complexity and the Frege-Geach Point", Synthese, vol. 198, pp. 3099-3130, <https://doi.org/10.1007/s11229-01902270-1>.

Conant, James, 2020, "Reply to Stroud on Kant and Frege: On the Relation of Thought to Judgment", en Sofia Miguens (comp.), The Logical Alien. Conant and His Critics, Harvard University Press, Cambridge, Massachusetts, pp. 783-829, <https://doi.org/10.4159/9780674242821>.

Correia, Fabrice, 2013, "Metaphysical Grounds and Essence", en Miguel Hoeltje, Benjamin Schnieder y Alex Steinberg (comps.), Varieties of Dependence. Ontological Dependence, Grounding, Supervenience, Response-Dependence, Philosophia Verlag, Múnich, pp. 271-292.

Bliss, Ricki y Kelly Trogdon, 2016, "Metaphysical Grounding", en Edward N. Zalta (ed.), The Stanford Encyclopedia of Philosophy, <https://plato.stanford.edu/entries/grounding/ >.

Hanks, Peter W., 2007, "How Wittgenstein Defeated Russell's Multiple Relation Theory of Judgment", Synthese, vol. 154, no. 1, pp. 121-146, < https://doi. org/10.1007/s11229-005-0195-y>.

Hanks, Peter W., 2011, "Structured Propositions as Types", Mind, vol. 120, no. 477, pp. 11-52, <https://doi.org/10.1093/mind/fzr011>.

${ }^{23}$ Adviértase que la mención sería ineliminable incluso si rechazar producir un ejemplar de la proposición < Lara es gris $>$ consistiese en producir un ejemplar de otra proposición en la que < Lara es gris $>$ fuese objeto de predicación.

"Este artículo fue escrito gracias al apoyo otorgado por el Gobierno de Chile mediante el proyecto Fondecyt no. 1200002. Me he beneficiado de las preguntas y observaciones de José Tomás Alvarado, Sebastián Briceño y Kênio Estrela, así como de los asistentes a una presentación de mi trabajo, una sesión muy provechosa del Seminario Web del grupo de Conceptos y Percepción, Universidad Nacional de Córdoba (Argentina); me refiero, en particular, a Mariela Aguilera, Andrés Crelier, Laura Danón, Daniel Kalpokas, Mauro Zapata y Ezequiel Zerbudis. Expreso también mi gratitud por los comentarios de dos árbitros anónimos de la revista. 
Hanks, Peter W., 2013, "What are the Primary Bearers of Truth?", Canadian Journal of Philosophy, vol. 43, nos. 5-6, pp. 558-574, <https://doi.org/10. 1080/00455091.2013.870723>.

Hanks, Peter W., 2015, Propositional Content, Oxford University Press, Oxford, <https://doi.org/10.1093/acprof:oso/9780199684892.001.0001>.

Jubien, Michael, 2001, "Propositions and the Objects of Thought", Philosophical Studies, vol. 104, no. 1, pp. 47-62, <https://doi.org/10.1007/0-30648134-0_10>.

King, Jeffrey C., Scott Soames y Jeff Speaks, 2014, New Thinking about Propositions, Oxford University Press, Oxford, <https://doi.org/10.1093/acprof: oso/9780199693764.001.0001>.

Lebens, Samuel, 2017, Bertrand Russell and the Nature of Propositions, Routledge, Nueva York, <https://doi.org/10.4324/9781315185361>.

Moltmann, Friederike, 2003, "Propositional Attitudes without Propositions", Synthese, vol. 135, no. 1, pp. 77-118.

Peacocke, Christopher, 2008, Truly Understood, Oxford University Press, Oxford, <https://doi.org/10.1093/acprof:oso/9780199239443.001.0001>.

Prior, Arthur Norman, 1971, Objects of Thought, Oxford University Press, Oxford, <https://doi.org/10.1093/acprof:oso/9780198243540.001.0001>.

Reiland, Indrek, 2013, "Propositional Attitudes and Mental Acts", Thought, vol. 1, no. 3, pp. 239-245, <https://doi.org/10.1002/tht3.42>.

Reiland, Indrek, 2019, "Predication and the Frege-Geach Problem", Philosophical Studies, vol. 176, no. 1, pp. 141-159, <https://doi.org/10.1007/ s11098-017-1009-z>.

Russell, Bertrand, 1912, The Problems of Philosophy, Oxford University Press, Oxford [edición de 1997].

Russell, Bertrand, 1913, Theory of Knowledge, Routledge, Londres [edición de 1992], <https://doi.org/10.4324/9780203713938>.

Sainsbury, Mark, 2002, "How can Some Thing Say Something?", en Mark Sainsbury, Departing from Frege, Routledge, Londres, pp. 102-111, <https://doi. org/10.4324/9780203218938>.

Schmitz, Michael, (en preparación), "Force, Content and the Varieties of Unity", en Michael Schmitz y Gabriele Mras (comps.), Force, Content and the Unity of Proposition, Routledge, Nueva York.

Soames, Scott, 2014a, "Cognitive Propositions", en King, Soames y Speaks, 2014, pp. 91-124, <https://doi.org/10.1093/acprof:oso/9780199693764. $003.0006>$.

Soames, Scott, 2014b, "Clarifying and Improving the Cognitive Theory", en King, Soames y Speaks, 2014, pp. 226-244, <https://doi.org/10.1093/acprof:oso/9780199693764.003.0012>.

Soames, Scott, 2015, Rethinking Language, Mind, and Meaning, Princeton University Press, Princeton, <https://doi.org/10.1515/9781400866335>.

Speaks, Jeff, 2014, "Representational Entities and Representational Acts", en King, Soames y Speaks, 2014, pp. 147-165, <https://doi.org/10.1093/acprof:oso/9780199693764.003.0008>. 
Trueman, Robert, 2021, Properties and Propositions. The Metaphysics of Higher-Order Logic, Cambridge University Press, Cambridge, <https://doi. org/10.1017/9781108886123>.

Recibido el 17 de enero de 2021; revisado el 29 de marzo de 2021; aceptado el 15 de mayo de 2021. 